\title{
Cyclical interactions with alliance-specific heterogeneous invasion rates
}

\author{
Matjaž Perc ${ }^{\star}$, Attila Szolnoki ${ }^{\dagger}$, and György Szabó ${ }^{\dagger}$ \\ ${ }^{\star}$ Department of Physics, Faculty of Education, University of Maribor, Koroška cesta 160, SI-2000 Maribor, Slovenia \\ ${ }^{\dagger}$ Research Institute for Technical Physics and Materials Science, P.O. Box 49, H-1525 Budapest, Hungary
}

\begin{abstract}
We study a six-species Lotka-Volterra type system on different two-dimensional lattices when each species has two superior and two inferior partners. The invasion rates from predator sites to a randomly chosen neighboring prey's site depend on the predator-prey pair, whereby cyclic symmetries within the two three-species defensive alliances are conserved. Monte Carlo simulations reveal an unexpected non-monotonous dependence of alliance survival on the difference of alliance-specific invasion rates. This behavior is qualitatively reproduced by a four-point mean-field approximation. The study addresses fundamental problems of stability for the competition of two defensive alliances and thus has important implications in natural and social sciences.
\end{abstract}

PACS numbers: 02.50.Le, 87.23.Ge, 89.75.Fb

Cyclical interactions are simple yet fascinating and powerful examples of evolutionary processes [1], able to provide insights into the intriguing mechanisms of Darwinian selection [2] as well as structural complexity [3] and pre-biotic evolution [4]. The simplest non-trivial food web describing such cyclical interactions is formed by three species that have relationships analogous to the well-known rock-scissors-paper (RSP) game, where strategies form a closed loop of dominance. Real-life examples of such interactions include the mating strategy of side-blotched lizards [5], overgrowths by marine sessile organisms [6], and competition among different strains of bacteriocin-producing bacteria [7]. Cycles are also common in the context of evolutionary game theory [8], where strategic complexity [9, 10] often leads to RSP type of dominance between different strategies [11].

Several theoretical aspects of multi-species cyclical dominance have already been studied in detail. For example, it has been established that three species in a cyclic dominance exhibit self-organizing behavior on the spatial grid [12, 13], whereby similar observations can be made also for system that incorporate more than three species, provided their total number does not exceed fourteen [14]. Phase transitions and selection have also been studied in the predator-prey models allowing motion throughout inhabitable vacant sites [15, 16]. Especially reticulate six-species models with mutation [17] and local mixing [18] have recently been studied rigorously, reporting the spontaneous emergence of defensive alliances and numerous stable spatial distributions as well as pertaining phase transitions in dependence on the topology of underlying food webs. In these systems the number of possible stationary states increases rapidly with the number of species because the solutions of subsystems (some species are missing) are also solutions of the whole system. The final stationary state can be determined by the competition of subsystem solutions (defensive alliances) that are characterized by their composition and spatio-temporal distribution of species. Consequently, the understanding of systems with many species requires the systematic analysis of all their subsystems. In the present work we will study a predator-prey model that can be considered as a six-species subsystem of strains of bacteria using two types of toxins and anti-toxins in their warfare [7].

Besides increasing the number of species, the complexity of models can be enhanced also by the introduction of hetero-

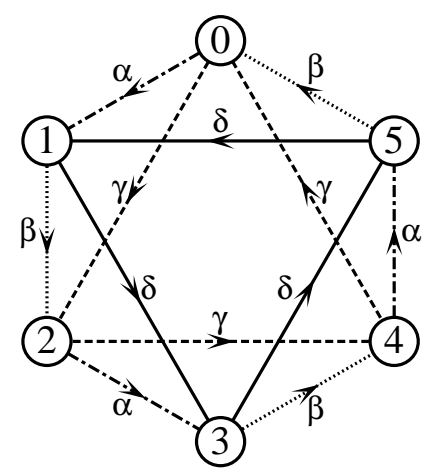

FIG. 1: Food web of the studied predator-prey model. Arrows point from predators towards prey with heterogeneous invasion rates specified along the edges.

geneous invasion rates between interacting individuals. Differences in invasion rates might affect the proportions of participating species in the habitat [19] as well as geometrical features of patterns on the spatial grid [20]. Moreover, it has recently been discovered that multi-species models of cyclical interactions comprising an even number of individuals are very sensitive to the independent variation of invasion rates [21]. The introduction of heterogeneous invasion rates also raises questions about the relevance of defensive alliances.

Presently, we thus study a reticulate six-species predatorprey model where each site $i$ of the square lattice is occupied by an individual belonging to one of the six species. Their corresponding distribution is given by a set of site variables $s_{i}=0, \ldots, 5$. The predator-prey relations and the corresponding invasion rates $(0<\alpha, \beta, \gamma, \delta<1)$ are defined by the food web presented in Fig. 11 For this choice of parameterization the two subsystems consisting of odd and even labeled species are equivalent to the thoroughly studied rock-scissorspaper game and the system remains unchanged under cyclic permutation ( $s \rightarrow s+2$ modulo 6 ) of species.

In case of homogeneous rates $(\alpha=\beta=\gamma=\delta=1)$ the system has two equivalent three-species states [denoted by $(0+2+4)$ and $(1+3+5)]$ exhibiting a self-organizing pattern maintained by cyclic invasions. These state are called defensive alliances because their members protect each other 
cyclically against the external invaders $[17,18]$. Consider for example the case when the species 0 invades allies $(1+3+5)$, in particular by attacking species 1 . Its intention is immediately disabled by the species 5 that is superior to both 0 and 1 . Thus, the intruder 0 is quickly abolished from the $(1+3+5)$ domain by the very same species 5 that dominates species 1 within the alliance. The same reasoning applies for all other possible attempts of non-allied species to invade a defensive alliance. Importantly, in this mechanism the proper spatiotemporal distribution of species plays a crucial role (the meanfield approximation cannot reproduce this feature).

By introducing alliance-specific heterogeneities in the invasion rates, we are capable of analyzing the competition between the defensive alliances. For example, we can study what happens when one of the associations is more aggressive towards the other $(\alpha \neq \beta)$ or when the internal mechanism fails to assure flawless protection against the invaders $(\gamma \neq \delta)$. In order to address these two issues systematically, it appears reasonable to introduce two parameters that, due to symmetries in the food web, uniquely determine the stationary state of the system. Particularly, let $G=\beta-\alpha$ and $H=\gamma-\delta$ where $H, G \in[-1,1]$. Note that the system behavior becomes trivial in two quadrants of the $H-G$ parameter space because the favored defensive alliance is supported by both mentioned mechanisms, thus leading to its undisputed dominance on the spatial grid. If $G<0(\beta<\alpha)$ and $H>0$ $(\gamma>\delta)$ allies $(0+2+4)$ receive a two-fold advantage. First, due to $\beta<\alpha(0+2+4)$ invade non-members faster than individuals in the competing alliance. Second, due to $\gamma>\delta$ the internal invasions within $(0+2+4)$ are faster than those in $(1+3+5)$, whereby faster internal cyclic invasions uphold a more effective protection shield against the external invaders by assuring a prompt response to a potential attack. An interesting competition emerges only if $G>0$ and $H>0$ (or equivalently if $G<0$ and $H<0$ ), which we are going to explore next. Due to the symmetry of the problem our analysis will be constrained to the parameter space spanning over $H, G \in[0,1]$.

We perform Monte Carlo (MC) simulations of the introduced six-species cyclical interaction model on the $L \times L$ spatial grid. Initially the six species are randomly distributed. The elementary steps are the following. First, two nearest neighbors are chosen at random, and second, if the two neighboring species form a predator-prey pair (species connected by an arrow in Fig. (1) the prey is killed with the rate specified along the arrow and an offspring of the predator occupies the prey's site. On the other hand, if the two randomly chosen species form a neutral pair (species not connected by an arrow in Fig. 1), or if both are identical, the second step dictates no action (nothing happens) and the MC simulation proceeds with executing step one. In accordance with the random sequential update, each individual is selected once on average during a particular Monte Carlo step (MCS). In order to characterize the stationary state we define the order parameter $m=\rho_{1}+\rho_{3}+\rho_{5}-\rho_{0}-\rho_{2}-\rho_{4}$, whereby $\rho_{s}$ $(s=0, \ldots, 5)$ denotes the fraction of species $s$ on the spatial grid. Here $m=1$ corresponds to the complete dominance of the alliance $(1+3+5)$. On the other hand, $m=-1$ indicates

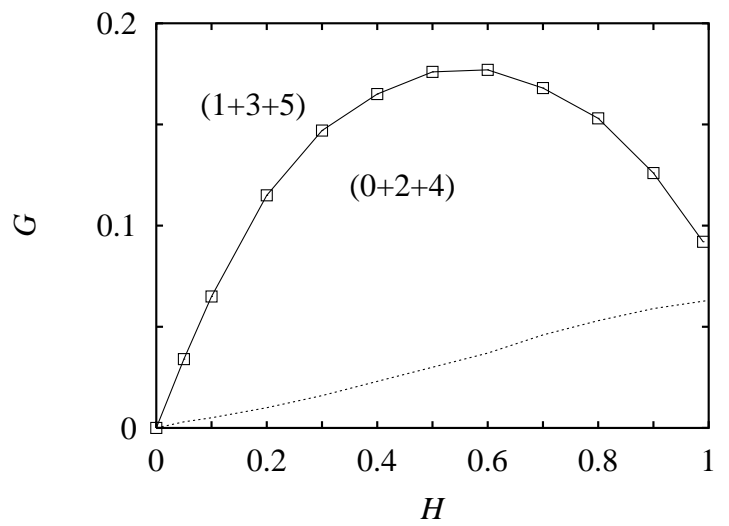

FIG. 2: Phase separation line dividing the two pure phases, characterized either by the exclusive dominance of the alliance $(0+2+4)$ or $(1+3+5)$, in dependence on $H$ and $G(\beta=\gamma=1)$. Linked squares were obtained by MC simulations while the dashed line results from the four-point cluster mean-field approximation.

the absolute authority of the association $(0+2+4)$.

We start by setting $G$ and $H$ equal to zero, whereby both alliances have an equal chance of eventually dominating the spatial grid via a domain growing process. In accordance with the above discussion of the studied model, it is reasonable to expect that as soon as $G$ rises above zero (keeping $H=0$ ) allies $(1+3+5)$ are favored as their members invade non-allied species more successfully $(\alpha<\beta)$. Thus, $m=1$ in the stationary state, meaning that the system evolves into the threespecies self-organizing phase $(1+3+5)$ as soon as members of $(0+2+4)$ die out. However, the advantage of $(1+3+5)$ given by $G>0$ can be compensated by choosing sufficiently large values of $H$, as shown in Fig. 2. As argued above, if $H$ rises above zero the internal invasions within allies $(1+3+5)$ slow down in comparison to $(0+2+4)$, thus decreasing the effectiveness of the protection of the odd alliance and in turn nullifying its advantage given by $G>0$.

Strikingly though, results of MC simulations shown in Fig. 22 reveal a non-monotonous phase diagram in dependence on $H$. In particular, the advantage of allies $(1+3+5)$ again increases if $H$ approaches 1 [the internal invasion rate $(\delta)$ vanishes]. As $\delta \rightarrow 0$ allies $(1+3+5)$ essentially stop to invade each other within the alliance. In this case $(\delta=0)$ an external species (e.g. 2) can survive in the bulk of its neutral pair (e.g. in the domain of species 5). As a result, the order parameter $m$ remains below 1 forming a frozen state after achieving dominance. The mechanism behind this interesting phenomenon is subtle and cannot be grasped at a glance. The above analysis was made also for some other fixed values of $\beta$ and $\gamma$ yielding essentially identical results.

Evidently, the mean-field analysis can be applied as an analytical tool to study the behavior of the proposed predator-prey model. Unfortunately, the resulting master equations fail to confirm above results. Namely, the solution for the fractions of species predicts a total dominance of allies $(1+3+5)$ if $G>0$ irrespective of $H$. This shortage of the classical mean-field approximation may be eliminated by applying the 
extended versions of dynamical mean-field theory that proved to be very appropriate for obtaining qualitatively correct phase diagrams for several non-equilibrium systems. The improved approach involves finding a hierarchy of evolution equations for the configurational probabilities on $k$-site clusters, where $k$ characterizes the level of approximation (for details, see e.g. [22, 23]). Nonetheless, the application of the method at the two-point level is still unable to account for the incursion of allies $(0+2+4)$ into the $G>0$ region if $H>0$. The first level that supports our above conjectures is the four-point level, of which the solution is displayed in Fig. 2] The fact that results of MC simulations differ from the four-point cluster mean-field approximation suggests that the unexpected nonmonotonous dependence is heavily routed in the short-range correlation of spatial distribution, which cannot be captured adequately by the ansatz of a four-point level approximation.

To obtain a better understanding of the spatial dynamics behind the phase diagram in Fig. 2 we examine the nature of phase transitions between $m=-1$ and $m=1$. Although the two competing effects of parameters $G$ and $H$ suggest that indeed a fine-tuning towards a stable co-existence of both alliances may be possible, thus assuring a rich diversity of species on the spatial grid, the reality is quite different. In fact, results in Fig. 2 suggest that phase transitions between the two pure phases are extremely sharp, and thus a stable co-existence of the two defensive alliances on the spatial grid is not feasible. Although we have only numerical arguments the extensive calculations performed in order to come to this conclusion leave extremely little room for alternatives.

Finally, it is instructive to examine the temporal evolution of the order parameter in the close vicinity of the phase boundary. Figure 3 illustrates that, at the beginning, the fraction of even labeled species decreases on both sides of the phase separation line. However, while above the phase boundary members of the even alliance go extinct, below the phase boundary allies $(0+2+4)$ are able to fully recover although only a minute portion (as little as 0.005 ) of the spatial grid is occupied by its members, eventually yielding $m=-1$. We argue that this remarkable and unusual behavior, which is intimately linked also with the non-monotonous phase diagram presented in Fig. 2, is related to the formation of a suitable boundary layer that modifies the interaction between both defensive alliances and ultimately tosses the dominance in favor of either $(1+3+5)$ or $(0+2+4)$. It is remarkable that a similar temporal evolution was reported in a group selection model [24] where the altruistic species almost reach extinction before taking over the population. However, as described above, in our case the spatiality is a fundamental ingredient since the recovery of the even alliance, occurring due to its dynamical benefit, is an interface driven domain growing process.

To confirm the importance of the boundary layer between both defensive alliances, we perform a stability analysis of the interface via MC simulations of the system with specially prepared initial conditions. In particular, we start the simulations with sharp boundaries that separate regions of initially randomly distributed even and odd labeled species. Within a $3200 \times 3200$ spatial grid we were able to set up 64 such straight boundary layers (without them interfering with each

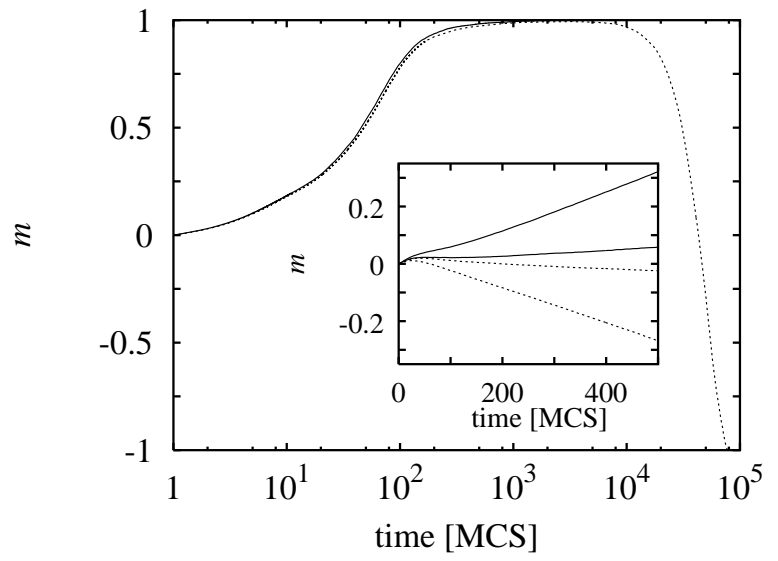

FIG. 3: Temporal evolution of the order parameter for $H=0.2$ and different values of $G$. Solid line depicts the temporal evolution obtained for $G=0.116$, while the dashed line corresponds to $G=0.114$. Inset shows the evolution of the order parameter from prepared initial states (see text for details). Lines in the inset correspond to $G=0.130,0.117,0.113$ and 0.110 from top to bottom.

other) and monitored in which direction the interface moved in dependence on $G$ placing the system below or above the phase boundary shown in Fig. 2 Additionally, results were averaged over 10 consecutive runs to minimize unwanted fluctuations. The inset in Fig. 3 shows the results for two values of $G$ below and two above the phase separation line. Note that $m$ measures the difference of areas on both sides of the initially sharp boundary between even and odd labeled species, thus uniquely determining also its spreading direction. Evidently, if $G$ is set above the phase separation line in Fig. 2 the boundary layer moves towards the area of $(0+2+4)$, thus foretelling an imminent dominance of $(1+3+5)$. Conversely, if $G$ is set below the phase separation line the boundary layer moves towards $(1+3+5)$, marking the advent of dominance of $(0+2+4)$ although the total fraction of its members on the spatial grid at that time might be minute. Hence, if the evolution of the system is initialized from a completely random initial distribution of species on the spatial grid, members of $(1+3+5)$ can utilize their advantage given by $G>0$ and rapidly start their conquest. However, as time goes by the seemingly defeated $(0+2+4)$ may self-organize into small clusters on the spatial grid, and if $G$ and $H$ are set appropriately, start to win back lost ground via growth along the interfaces, as explained above and presented in Fig. 3 .

In order to check the robustness of the above-described behavior the present predator-prey model was also studied on the honeycomb and triangular lattices having different coordination numbers. Figure 4 clearly shows that the honeycomb lattice additionally pronounces the non-monotonous variation of the phase boundary. Conversely, the incursion of allies $(0+2+4)$ is less emphasized by the triangular lattice, which may be related to the increased coordination number, bringing the MC simulations closer to the mean-field behavior. Results presented in Fig. 4 further stress the importance of spatiality and with it related distribution of species and resulting bound- 


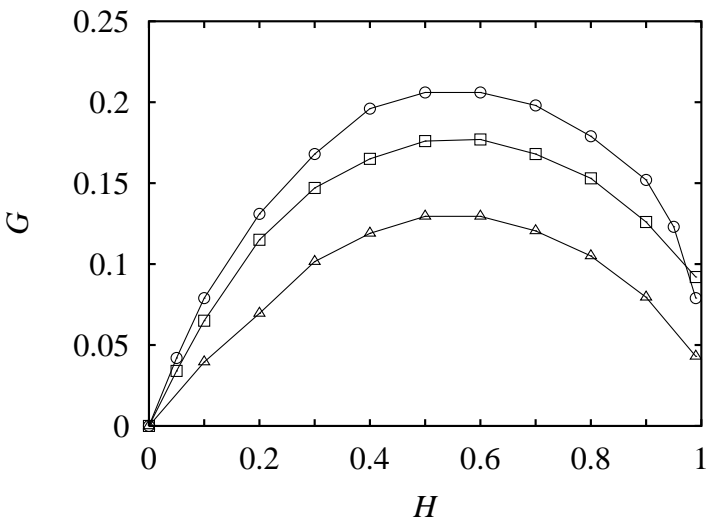

FIG. 4: Phase boundaries between the stationary states $(0+2+4)$ and $(1+3+5)$ for different lattice structures. Linked symbols depict phase separation lines for the honeycomb (circles), square (squares), and triangle (triangles) lattice.

ary layers among defensive alliances on the spatial grid.

In sum, we have studied a six-species predator prey model with special heterogeneous invasion rates that were introduced in accordance with the two spontaneously emerging defensive alliances. We have shown that an increased aggression to- wards non-allied species could be tamed by decreasing the willingness of members to attack individuals within the alliance itself. Remarkably though, if individuals completely sized to invade its own allied members the benefit of increased aggression towards non-allied species again increased. These two facts resulted in a non-monotonous dependence of alliance survival on the difference of alliance-specific invasion rates, which we attributed to the underlying spatial dynamics of the system. We also discovered that despite the ability of fine-tuning two system parameters, a stable state enabling the co-existence of both defensive alliances on the spatial grid is not possible, thus resulting in sharp phase transitions between the two absorbing states. However, the newly introduced alliance-specific heterogeneous invasion rates might yet prove valuable by discovering new ways of assuring biodiversity, either within generalized Lotka-Volterra models [25], via the impact of stochasticity [26], or oscillatory mechanisms [27]. We hope that the study will prove vital for the understanding of the robustness of alliance formation, their competition, and for the effect of spatial structures on the evolution of food webs in real systems, which appear to range from strains of bacteria to man made economic systems.

This work was supported by the Hungarian National Research Fund (T-47003), the European Science Foundation (COST P10), and the Slovenian Research Agency (Z1-9629).
[1] J. Hofbauer and K. Sigmund, Evolutionary Games and Population Dynamics (Cambridge University Press, Cambridge, 1998).

[2] J. Maynard Smith and G. R. Price, Nature 246, 15 (1973).

[3] A. S. Watt, J. Ecol. 35, 1 (1947).

[4] S. Rasmussen, L. Chen, D. Deamer, D. C. Krakauer, N. H. Packard, P. F. Stadler, and M. A. Bedau, Science 303, 963 (2004).

[5] B. Sinervo and C. M. Lively, Nature 380, 240 (1996).

[6] M. T. Burrows and S. J. Hawkins, Mar. Ecol. Prog 167, 1 (1998).

[7] G. Szabó and T. Czárán, Phys. Rev. E 63, 061904 (2001).

[8] M. A. Nowak, Evolutionary Dynamics: Exploring the Equations of Life (Harward University Press, Harvard, 2006).

[9] C. Hauert, S. De Monte, J. Hofbauer, and K. Sigmund, Science 296, 1129 (2002).

[10] A. Traulsen and J. C. Claussen, Phys. Rev. E 70, 046128 (2004).

[11] D. Semmann, H.-J. Krambeck, and M. Milinski, Nature 425, 390 (2003).

[12] K. Tainaka, Phys. Rev. Lett. 63, 2688 (1989).

[13] L. Frachebourg, P. L. Krapivsky, and E. Ben-Naim, Phys. Rev. E 54, 6186 (1996).
[14] L. Frachebourg and P. L. Krapivsky, J. Phys. A 31, L287 (1998).

[15] G. Szabó and G. A. Sznaider, Phys. Rev. E 69, 031911 (2004).

[16] M. He, Y. Cai, Z. Wang, and Q.-H. Pan, Int. J. Mod. Phys. C 16, 1861 (2005).

[17] G. Szabó and T. Czárán, Phys. Rev. E 64, 042902 (2001).

[18] G. Szabó, J. Phys. A: Math. Gen. 38, 6689 (2005).

[19] M. Frean and E. D. Abraham, Proc. R. Soc. Lond. B 268, 1 (2001).

[20] G. Szabó and A. Szolnoki, Phys. Rev. E 65, 036115 (2002).

[21] K. Sato, N. Yoshida, and N. Konno, Appl. Math. Comp. 126, 255 (2002).

[22] R. Dickman, Phys. Lett. A 122, 463 (1987).

[23] G. Szabó and G. Fáth (2006), arXiv:cond-mat/0607344.

[24] A. T. C. Silva and J. F. Fontanari, Eur. Phys. J. B 7, 385 (1999).

[25] O. Malcai, O. Biham, P. Richmond, and S. Solomon, Phys. Rev. E 66, 031102 (2002).

[26] T. Reichenbach, M. Mobilia, and E. Frey, Phys. Rev. E 74, 051907 (2006).

[27] M. Kowalik, A. Lipowski, and A. L. Ferreira, Phys. Rev. E 66, 066107 (2002). 